\title{
CONTROLE DO ÁCARO DA FALSA FERRUGEM (Phyllocoptruta oleivora Ashm., 1879) POR UM JUVENÓIDE E OUTROS DEFENSIVOS AGRÍCOLAS
}

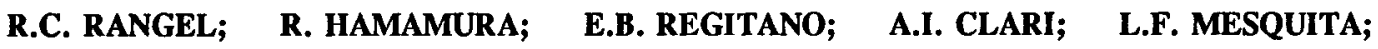 \\ M.A.C. CARDOSO; \\ Dep. de Zoologia da ESALO/USP, Caixa Postal, 9 - CEP: 13418-900-Piracicaba,SP. \\ F.A.M. MARICONI \\ Prof. Titular do Dep. de Zoologia da ESALQ/USP, Caixa Postal, 9 - CEP: 13418-900-Piracicaba,SP.
}

\begin{abstract}
RESUMO: Vários defensivos agrícolas, incluindo um juvenóide, foram aplicados contra o ácaro da falsa ferrugem em pomar localizado em Limeira,SP, uma das principais áreas citricolas do Brasil. Os tratamentos constaram de uma única aplicação, feita em 01/10/89. Tratamentos: A) testemunha; B) flucicloxurom (juven6ide) $40 \mathrm{~cm}^{3}$; C) bromopropilato $65 \mathrm{~cm}^{3}$; D) quinometionato + enxofre $200 \mathrm{~cm}^{3}$; E) quinometionato + enxofre $250 \mathrm{~cm}^{3}$; F) formetanato $22,5 \mathrm{~g}$; G) formetanato $31,5 \mathrm{~g}$. As quantidades referem-se aos produtos comerciais para 100 litros de água. Cada laranjeira recebeu 10 litros de calda. Espalhante-adesivo: "Extravon" (25 $\mathrm{cm}^{3} / 100$ litros). 0 delineamento estatístico feito foi de blocos ao acaso, com 4 repetições. Foram feitas 6 avaliações: 1 prévia e 5 ap6s 06, 20, 36, 50 e 68 dias da aplicação. F, G e C foram os melhores tratamentos. D e E foram eficientes somente aos 06 e 20 dias e o juvenóide, aos 20, 36 e 50 dias.
\end{abstract}

Descritores: ácaro da falsa ferrugem, citros, Phyllocoptrula oleivora, juvenoide.

\section{CONTROL OF CITRUS RUST MITE (Phyllocoptrute oleivore Ashm., 1879) BY A JUVENOID AND OTIER PESTICIDES}

\begin{abstract}
In order to control the citrus rust mite on orange-trees a field experiment was carried out in Limeira, State of São Paulo, Brazil, an important citrus producing area. Treatments correspondend to a single application on 01/10/89 of: A) check; B) juvenoid flucycloxuron, $40 \mathrm{~cm}^{3}$; C) bromopropilate $65 \mathrm{~cm}^{3}$; D) quinomethionate + sulphur $200 \mathrm{~cm}^{3}$; E) quinomethionate + sulphur $250 \mathrm{~cm}^{3}$; F) formetanate $22,5 \mathrm{~g}$; G) formetanate $31,5 \mathrm{~g}$. The quantities are of commercial formulations in 100 liters of water. Each tree received 10 liters of the mixtures. Avaliations were made before spraying and 6, 20,36,50 and 68 days after the application. The most effective results in the last three avaliations were obtained with treatments $F, G$ and $C$. Treatments $D$ and $E$ were effective only for 6 and 20 days and the juvenoid for 20,36 and 50 days after spraying.
\end{abstract}

Key Words: citrus rust mite, Phyllocoptruta oleivora, citrus, juvenoid.

\section{INTRODUÇÃO}

Dada a importância atual da citricultura no Brasil, o ácaro da falsa ferrugem Phyllocoptruta oleivora (Ashm., 1879) assume grande destaque e sendo uma das principais pragas justifica-se que trabalhos de combate estejam sendo continuamente realizados.

Mortalidades superiores a $91 \%$ foram obtidas por MURAI et al. (1973) com a pulverização de bromopropilato, clorobenzilato, ometoato e dicrotofós, aos 41 dias de aplicação. NAKANO et al. (1977) obtiveram melhores resultados com o óxido de fembutatina, dicrotofós + malatiom e clorobenzilato. SANTOS et al. (1977) obtiveram reduções notáveis na população com o aldicarbe granulado a $10 \%$, no solo, à razão de 100 e $200 \mathrm{~g}$ por laranjeira; não houve diferença significativa entre as duas dosagens, até 66 dias após a aplicação. BRUNELLI et al. (1978) conseguiram resultados de combate surpreendentes com o aldicarbe e o oxamil, ambos granulados incorporados ao solo. A partir de 126 dias o oxamil começou a apresentar controle menos eficiente. $O$ aldicarbe atuou muito bem até aos 218 dias da aplicação (4g IA/planta) e $10 \mathrm{~g}$ IA/planta até aos 246 dias. MARCONATO et al. (1980) obtiveram bons resultados com a pulverização de ciexatina e triflumurom (ex-triflurom), e no solo, com granulados de aldicarbe e oxamil. $O$ bromopropilato foi considerado por ALMEIDA et al. (1981) muito eficiente, embora tenha caído uma forte e prolongada chuva logo após a pulverização realizada por eles; por outro lado, esses autores 
não recomendam os inseticidas-acaricidas piretróides FMC 54617 e bifentrina (FMC 54800) em citros, devido ao intenso desequilíbrio biológico causado. MARICONI et al (1986) aplicaram diversos defensivos, entre eles, o bromopropilato e a clofentezina. Estes acaricidas conduziram a resultados bons a excelentes até 117 dias de pulverização. GERALDI et al. (1987) experimentaram granulados sistêmicos no solo e líquidos em pulverização. Duas formulaçōes de dicofol foram muito eficientes: uma delas, formulada com dicofol téenıco não purificado, foi um pouco melhor, principalmente aos 83 dias da pulverização; a outra, preparada com dicofol livre de várias impurezas, atuou muito bem até aos $\mathbf{7 0}$ dias. O aldicarbe apresentou bons resultados; tornou-se muito bom aos 53, 70 c 83 dias, após a ocorrência de chuvas.

\section{MATERIAL E MÉTODOS}

Local Campo experumental instalado no Síto Santa Rosa, municípıo de Limeira, Estado de São Paulo, de propriedade do Sr Etelvino Novello.

Campo Experimental. Formado de laranjeiras da variedade Pera Natal, com idade de 9 anos, plantadas em espaçamentos de $6,30 \mathrm{~m}$ entre plantas e 7,10m entre linhas (222 plantas por hectare). As árvores apresentavam altura média de $3,5 \mathrm{~m}$.

Tratamentos: Em número de 7, com quatro repetições, tendo sido adotado o delineamento de blocos casualizados. Cada parcela cra constituída de três árvores, sendo que somente a árvore central foi pulverizada. Os tratamentos, produtos comerciais, formulações e consumo de materiais podem ser vistos na Figura 1.

Aplicação. Uma únıca pulverização foi realizada em 01 de outubro de 1989. Em todos os tratamentos os produtos foram aplicados com pulverizadores costais motorizados. Foram utilizados 10 litros de calda por planta.

Coletas: Foram realizadas 06 amostragens: a inicial em 28 de setembro de 1989 (03 dias antes da aplicação) e cinco outras, 6, 20, 36 e 50 e 68 dias após a aplicação. Em cada amostragem foram apanhadas 30 folhas por planta (30 folhas por parcela; 120 folhas por tratamento). A colcta das folhas foi feita ao acaso em toda a volta das plantas, com a ressalva de que não fossem muito velhas ou muito novas (com coloração intermediária). As folhas de cada parcela eram postas em saquinhos plásticos rotulados $\mathrm{e}$, em seguida, em caixas de isopor. Estas foram protegidas do sol e levadas para o laboratório logo após o término da coleta.

Contagens: realizadas em laboratório, com o auxílio de microscópios estereoscópicos com aumento de 30 vezes. Numa área circular, feita com vazador $\mathrm{n}^{\circ} 12$ (18 $\mathrm{mm}$ de diâmetro interno), contaram-se todos os ácaros presentes. A área localizava-se na página inferior da folha, próxima a sua base (TABELA 1).

Reduçāo real ou eficiência: Calculada pela fórmula de "Abbott", tomando-se por base a população de ácaros de cada tratamento, transformada em porcentagem de sobrevivência (TABELA 2).

Análise estatística: $O$ número de ácaro das 4 parcelas (TABELA 3), em todos os tratamentos e em cada avaliação, foi transformado em raiz quadrada de $x+0,5$, sendo $\underline{x}$ a quantidade de ácaro $\mathrm{cm}$ cada parcela. A soma dos 4 valores transformados foi dividida por 4, para se ter a população média do tratamento numa determinada avaliação.

Os resultados foram analisados pelo teste "F" e, a seguir, fez-se a análise das médias pelo teste de "Tukey" ao nível de $5 \%$ de probabilidade (TABELA 4).

\section{RESULTADOS E DISCUSSÃO}

Pela análise estatística, a nível de $5 \%$ de probabilidade (TABELA 1) e mortalidades reais (TABELA 2), pode-se observar os seguintes resultados:

Contagem prévia: todos os tratamentos se assemelham à testemunha.

1a Contagem (após 06 dias da aplicação): todos os tratamentos diferiram de A (testemunha); B (juvenóide) foi menos eficiente, ao contrárı dos outros 5 .

$2^{\mathrm{a}}$ Contagem: (20 dias após): todos os tratamentos diferiram da testemunha. B melhorou e os demais não se diferenciaram entre si e continuaram a apresentar resultados excelentes. 


\begin{tabular}{|c|c|c|c|c|}
\hline TRATAMENTO & \multicolumn{2}{|c|}{ FORMULAÇÃO(*) } & & $\begin{array}{l}\text { FORMULAÇÃO (**) } \\
\text { (em } 100 \text { l de água) }\end{array}$ \\
\hline \multicolumn{5}{|l|}{ A - testemunha } \\
\hline B - flucicloxurom & Andalin 250 CE Uniroyal & CE $25 \%$ & & $40 \mathrm{~cm}^{3}$ \\
\hline C - bromopropilato(***) & Neoron $500 \mathrm{CE}$ & CE $50 \%$ & & $65 \mathrm{~cm}^{3}$ \\
\hline D - quinometionato + enxofre & Morestan + Enxofre & $\begin{array}{l}\mathrm{SC} \\
60 \%)\end{array}$ & + & $200 \mathrm{~cm}^{3}$ \\
\hline E - quinometionato + enxofre & Morestan + Enxofre & $\begin{array}{l}\mathrm{SC} \\
60 \%)\end{array}$ & + & $250 \mathrm{~cm}^{3}$ \\
\hline F - formetanato & Dicarzol 500 & P. Sol. $50 \%$ & & $22,5 \mathrm{~cm}^{3}$ \\
\hline G - formetanato & Dicarzol 500 & P. Sol. $50 \%$ & & $31,5 \mathrm{~cm}^{3}$ \\
\hline \multicolumn{5}{|c|}{$\begin{array}{l}\text { (*) Formulação comercial: CE (concentrado emulsionável), SC (suspensão concentrada) e P. Sol. (pó } \\
\text { solúvel). } \\
\text { A mistura (D e E) tem } 12 \% \text { de quinometionato }+60 \% \text { de enxofre. } \\
(* *) \text { Para } 100 \text { litros: "Extravon", } 25 \mathrm{~cm}^{3} \\
(* * *) \text { Tratamento Padrão. }\end{array}$} \\
\hline
\end{tabular}

Figura 1 - Quadro de tratamentos, formulações e consumo de material para o combate experimental ao "ácaro da falsa ferrugem" em citros.

TABELA 1 - População do "ácaro da falsa ferrugem" em cada tratamento e em cada avaliação (soma das 4 parcelas), durante o experimento.

\begin{tabular}{|c|c|c|c|c|c|c|}
\hline \multirow{2}{*}{$\begin{array}{l}\text { Trata- } \\
\text { mento }\end{array}$} & \multicolumn{2}{|c|}{ POPULAÇÃ } & DO ÁCAR & DA FALSA & \multicolumn{2}{|c|}{ FERRUGEM } \\
\hline & Prévia & $\begin{array}{c}\text { Após } 6 \\
\text { dias }\end{array}$ & $\begin{array}{c}\text { Após } 20 \\
\text { dias }\end{array}$ & $\begin{array}{c}\text { Após } 36 \\
\text { dias }\end{array}$ & $\begin{array}{c}\text { Após } 50 \\
\text { dias }\end{array}$ & $\begin{array}{c}\text { Após } 68 \\
\text { dias }\end{array}$ \\
\hline A & 1510 & 1176 & 377 & 87 & 44 & 10 \\
\hline B & 1601 & 405 & 60 & 10 & 4 & 4 \\
\hline C & 1501 & 32 & 13 & 12 & 5 & 1 \\
\hline D & 1559 & 38 & 32 & 83 & 37 & 3 \\
\hline E & 1526 & 34 & 28 & 81 & 64 & 8 \\
\hline $\mathbf{F}$ & 1560 & 58 & 18 & 2 & 3 & 0 \\
\hline G & 1575 & 31 & 6 & 5 & 1 & 1 \\
\hline
\end{tabular}

$3^{\mathrm{a}}$ e $4^{\mathrm{a}}$ Contagens $(36$ e 50 dias depois da pulverização): D e E (ambos, quinometionato + enxofre) sofreram drástica redução em suas eficiências. Os 4 melhores tratamentos $\mathrm{C}$ (bromopropilato), B (juvenóide), G (formetanato, dosagem maior) e $F$ (formetanato, dosagem menor) não se mostraram diferentes entre si. $F(98,3 \%$ e
$96,6 \%$ de eficiência) e G $(94,8 \%$ e $96,6 \%)$ foram os melhores.

$5^{\mathrm{a}}$ Contagem (depois de 68 dias): a população de ácaro foi muito reduzida; entretanto $C, G$ e $F$ continuaram diferentes da testemunha. A população de ácaros fitoseiídeos foi, antes da pulverização, 
TABELA 2 - Combate ao "ácaro da falsa ferrugern": redução real (eficiência) a diferentes intervalos e tratamentos.

\begin{tabular}{|c|c|c|c|c|c|}
\hline \multirow{2}{*}{$\begin{array}{l}\text { Trata- } \\
\text { mento }\end{array}$} & \multicolumn{3}{|c|}{ REDUÇ Ã O } & \multicolumn{2}{|c|}{$(\%) \quad(*)$} \\
\hline & $\begin{array}{c}\text { Após } 6 \\
\text { dias }\end{array}$ & $\begin{array}{c}\text { Após } 20 \\
\text { dias }\end{array}$ & $\begin{array}{c}\text { Após } 36 \\
\text { dias }\end{array}$ & $\begin{array}{c}\text { Após } 50 \\
\text { dias }\end{array}$ & $\begin{array}{c}\text { Após } 68 \\
\text { dias }\end{array}$ \\
\hline \multicolumn{6}{|l|}{ A } \\
\hline B & 67,5 & 85,2 & 89,7 & 93,1 & 57,1 \\
\hline $\mathrm{C}(* *)$ & 97,3 & 96,4 & 86,2 & 89,7 & 85,7 \\
\hline D & 96,9 & 91,6 & 8,6 & 17,2 & 71,4 \\
\hline $\mathrm{E}$ & 97,2 & 92,8 & 8.6 & 0,0 & 28,6 \\
\hline F & 95,3 & 95,2 & 98,3 & 96,6 & 100,0 \\
\hline G & 97,4 & 98,4 & 94,8 & 96,6 & 85,7 \\
\hline
\end{tabular}

(*) Pcla Fórmula transformada de Abbolt $\mathrm{RR}=[(\%$ sobrev. test. $-\%$ sobr. trat. $) / \%$ sobrev. test. $] \times 100$ sobrev = sobrevivência; test. = testemunha; trat. = tratamento

(**) Tratamento padrão.

TABELA 3 - Combate experımental ao "ácaro da falsa ferrugem" em citros. população de ácaro predador nos diversos tratamentos e diferentes intervalos (soma das 4 parcelas).

\begin{tabular}{cccccccc}
\hline \hline \multirow{2}{*}{ Trata- } & \multicolumn{2}{c}{ P O P U L A Ç Ã O } & D O & Á C A R o & \multicolumn{2}{c}{ P R E D A D O R } \\
\cline { 2 - 7 } mento & Prévia & $\begin{array}{c}\text { Após 6 } \\
\text { dias }\end{array}$ & $\begin{array}{c}\text { Após } 20 \\
\text { dias }\end{array}$ & $\begin{array}{c}\text { Após 36 } \\
\text { dias }\end{array}$ & $\begin{array}{c}\text { Após 50 } \\
\text { dias }\end{array}$ & $\begin{array}{c}\text { Após 68 } \\
\text { dias }\end{array}$ \\
\hline A & 5 & 4 & 17 & 4 & 9 & 1 \\
B & 5 & 0 & 3 & 1 & 8 & 0 \\
C & 6 & 0 & 2 & 0 & 3 & 0 \\
D & 2 & 0 & 0 & 4 & 16 & 2 \\
E & 4 & 0 & 0 & 7 & 4 & 2 \\
F & 5 & 0 & 1 & 4 & 8 & 3 \\
G & 3 & 0 & 0 & 3 & 5 & 2 \\
\hline \hline
\end{tabular}

muito baixa; em nenhuma avaliação, esses predadores apresentaram alta população.

\section{CONCLUSÕES}

O formetanato conduziu a excelentes resultados, sem mostrar diferenças significativas entre as duas dosagens, dos 06 aos 68 dias. $O$ bromopropilato apresentou excelentes a bons resultados. O juvenóide (Пlucicloxurom) foi bom dos 20 aos 50 dias.

As duas dosagens de quinometionato + enxofre foram excelentes a boas somente aos $06 \mathrm{e}$ 20 dias de pulverização. 
TABELA 4 - Combate experimental ao "ácaro da falsa ferrugem" em citros: médias das populações do ácaro, a diferentes intervalos, transformadas em raiz quadrada de $x+0,5$ e resultados estatísticos (Tukey, $5 \%)$.

\begin{tabular}{|c|c|c|c|c|c|c|c|}
\hline \multirow{2}{*}{$\begin{array}{l}\text { TRATA- } \\
\text { MENTO }\end{array}$} & \multicolumn{4}{|c|}{ RESULTADO } & \multicolumn{3}{|c|}{ ESTATISTICO } \\
\hline & Prévia & Após $6 \mathrm{~d}$ & dias & Ap6́s 20 dias & Após 36 dias & Ap6́s 50 dias & Após 68 dias \\
\hline A & 18,08 a & $17,15 \mathrm{a}$ & & 9,63 a & $4,62 \mathrm{a}$ & $3,37 a$ & $1,70 \mathrm{a}$ \\
\hline B & 19,48 a & 10,05 & $\mathrm{~b}$ & $3,81 \quad b$ & $1,64 \quad b$ & $1,18 \quad b$ & $1,13 \mathrm{a} \mathrm{b}$ \\
\hline $\mathrm{C}$ & $19,06 \mathrm{a}$ & 2,84 & c & $1,90 \quad \mathrm{~b} \mathrm{c}$ & $1,70 \mathrm{~b}$ & $1,27 \quad b$ & $0,84 \quad b$ \\
\hline $\mathrm{D}$ & $19,66 \mathbf{a}$ & 3,12 & c & $2,88 \quad \mathrm{~b} \mathrm{c}$ & $4,55 \mathrm{a}$ & $3,06 \mathrm{a}$ & $1,06 \mathrm{a} \mathrm{b}$ \\
\hline $\mathrm{E}$ & $19,36 \mathrm{a}$ & 2,86 & c & 2,49 b c & $4,34 \mathrm{a}$ & $3,99 \mathrm{a}$ & $1,48 \mathrm{a} \mathrm{b}$ \\
\hline $\mathrm{F}$ & $19,34 \mathrm{a}$ & 3,67 & c & $2,19 \mathrm{bc}$ & $0,97 \mathrm{~b}$ & $0,93 \quad b$ & $0,71 \quad b$ \\
\hline $\mathrm{G}$ & $19,63 \mathrm{a}$ & 2,84 & $\mathrm{c}$ & 1,32 & $1,19 \quad \mathrm{~b}$ & $0,84 \quad \mathrm{~b}$ & $0,84 \mathrm{a}$ \\
\hline C.V. (\%) & 25,12 & 15,08 & & 27,76 & 35,98 & 26,04 & 32,26 \\
\hline D.M.S. & 11,28 & 2,14 & & 2,24 & 2,28 & 1,27 & 0,83 \\
\hline
\end{tabular}

Médias seguidas da mesma letra não diferem estatisticamente entre si.

(*) Após a obtenção de raiz quadrada de $\mathrm{x}+0,5$, fez-se a soma dos resultados das 4 parcelas e dividiu-se tudo por 4.

\section{REFERÊNCIAS BIBLIOGRÁFICAS}

ALMEIDA, S.L.; CORTE, C.R.; MORAIS, A.A.; GALHARDO, L.C.S.; FEKETE, T.J.; MARICONI, F.A.M. Defensivos químicos e o fungo Hirsutella thompsonii (Fisher, 1950) pulverizados contra Phyllocoptruta oleivora (Ashm., 1879) (ácaro da falsa ferrugem dos citros. O Solo, Piracicaba, v.73, n.2, p.11-17, 1981.

BRUNELLI JUNIOR, H.C.; CARVALHO, J.C.; OLIVEIRA FILHO, J.C.; FAGAN, R.; SANTOS, B.M.; AMORIM NETO, L.A.; MARICONI, F.A.M Granulados sistêmicos incorporados ao solo no combate ao ácaro Phyllocoptruta oleivora (Ashm., 1879) em laranjeiras adultas. O Solo, Piracicaba, n.70, n.2, p.15-19, 1978.

GERALDI, F.I.; BIONDO, C.J.; CLARI, A.I.; DONATONI, J.L.; RAIZER, A.J.; ARASHIRO, F.Y.; MARICONI, F.A.M. Combate ao ácaro da falsa ferrugem Phyllocoptruta oleivora (Ashm, 1879), em laranjeiras com granulados sistêmicos e líquidos. O Solo, Piracicaba, v.79, n.1/2, p.27-32, 1987.

MARCONATO, J.R.; TAVARES, S.; BRUNELLI JUNIOR, H.C.; FAGAN, R.; OLIVEIRA FILHO, J.C.; CARVALHO, J.C.; MARICONI, F.A.M. Combate químico ao "ácaro da falsa ferrugem" Phyllocoptruta oleivora (Ashm., 1879). O Solo,
Piracicaba, v.72, n.1, p.53-56, 1980.

MARICONI, F.A.M.; MOTTA, R.; SILVA, J.M., TAKAOKA, M.; RAIZER, A.J.; KATO, W.Y. Eficiência no combate ao ácaro da falsa ferrugem Phyllocoptruta oleivora (Ashm, 1879). Anais da Escola Superior de $\Lambda$ gricultura "Luiz de Queiroz", Piracicaba, v.43, p.751-762, 1986.

MURAI, N.T.; IDAGAWA, T.; MARICONI, F.A.M Pulverização a alto volume contra o ácaro da falsa ferrugem dos citros Phyllocoptruta oleivora (Ashm., 1879). O Solo, Piracicaba, v.65, n.1, p.27-29, 1973.

NAKANO, O.; SANTOS, L.A.; SUGUINO, H.; ARRUDA, J.M.M. Controle químico experimental visando o "ácaro da falsa ferrugem" Phyllocoptrua olesvora (Ashm., 1879) em citros. Divulgação Agronônica, São Paulo, v.42, p.16-20, 1977.

SANTOS, B.M.; BRUNELLI JUNIOR, H.C.; FAGAN, R.; AMORIM NETO, L.A.; TARDIVO, J.C.; DIONÍSIO, A.; FRANCO, J.F.; MARICONI, F.A.M Combate experimental ao "ácaro da falsa ferrugem da laranjeira" com sistêmicos aplicados no solo. O Solo, Piracicaba, v.69, n.2, p.32-36, 1977.

Trabalho entregue para publicação em 08.11 .90

Trabalho aprovado para publicação em 30.09.92 